\title{
Mejorar la salud pública y los sistemas de salud mediante políticas fundamentadas en la evidencia en la Región de las Américas*
}

\author{
Tomás Pantoja 1 , Jorge Barreto ${ }^{2}$ y Ulysses Panisset ${ }^{3}$
}

\begin{abstract}
Traducir los conocimientos en políticas y prácticas puede mejorar la salud pública y los sistemas de salud en la Región de las Américas, señalan Tomás Pantoja y cols.
\end{abstract}

ר 1 enfoque de que la formulación de políticas debe fundamentarse en la evidencia -es decir, que las decisiones en cuanto a las políticas deben sustentarse en el uso sistemático y transparente de datos científicos- atrajo mucha atención internacional en los primeros años del milenio. El movimiento fue incentivado por el Informe mundial sobre el conocimiento orientado a mejorar la salud del 2004 y las declaraciones de las Cumbres Ministeriales sobre Investigación en Salud, celebradas en Ciudad de México en el 2005 y en Bamako (Malí) en el 2008 y convocadas ambas por la Organización Mundial de la Salud. ${ }^{12}$ De manera más reciente, en la Agenda 2030 para el Desarrollo Sostenible, de las Naciones Unidas, se presentaron 17 objetivos para el desarrollo mundial, en los cuales las políticas fundamentadas en la investigación desempeñarán un papel clave.

En este contexto, en el 2009 la Organización Panamericana de la Salud/ Organización Mundial de la Salud (OPS/ OMS) formuló su Política de investigación para la salud. ${ }^{3}$ En ella se subrayó tanto la necesidad de que las investigaciones que se realizan cumplan con normas mínimas de calidad como de que sean relevantes a fin de fundamentar las políticas de salud. También se describió el entorno necesario para producir investigaciones que tengan impacto: una gobernanza eficaz de la investigación y una fuerza laboral capacitada que trabaje en redes colaborativas. $^{3}$ El objetivo de la política de lograr un impacto positivo en la salud mediante la investigación depende de que se establezca un diálogo constructivo entre los diferentes interesados directos que financian, producen y usan las investigaciones. Esto implica empoderar a las organizaciones de la sociedad civil para que establezcan las prioridades en el ámbito de la investigación, y difundir los resultados de las investigaciones en formatos apropiados para los diferentes públicos.

\section{MENSAJES CLAVE}

- En la Región de las Américas, muchos responsables de formular políticas reconocen la importancia de usar la investigación para fundamentar las decisiones relativas a los sistemas de salud.

- Sin embargo, se acepta mucho más que los conocimientos se traduzcan en la formulación de políticas en la esfera clínica y de la salud pública que en el campo de los sistemas de salud.

- En la Región, el crecimiento y la consolidación de los sistemas nacionales de investigación en materia de salud para generar evidencia ha sido desigual y se ha basado en iniciativas aisladas de fortalecimiento de la capacidad.

- Algunos países han establecido una infraestructura formal para ejecutar iniciativas nacionales de traslación de la investigación.

- A pesar de ello, en la mayor parte de los casos las actividades se realizan por medio de proyectos puntuales que no cuentan con financiamiento sostenible más allá del ciclo de vida del proyecto en cuestión.

\footnotetext{
* Traducción oficial al español efectuada por la Organización Panamericana de la Salud. En caso de discrepancia, prevalecerá la versión original en inglés.
}

\section{Profesor adjunto, Departamento de Medicin Familiar, Pontificia Universidad Católica de Chile, Santiago, Chile. $₫$ tpantoja@med.puc.cl}

\footnotetext{
2 Investigador, Fundación Oswaldo Cruz (FIOCRUZ), Brasilia, Brasil.

3 Profesor adjunto, Facultad de Medicina, Universi-
} dad Federal de Minas Gerais, Brasil 
El propósito de este artículo es analizar los retos actuales en el ámbito de la investigación para la salud y describir las iniciativas exitosas que se han puesto en marcha en la Región de las Américas a fin de lograr un impacto positivo en la salud de la población.

\section{PRINCIPALES RETOS}

Los investigadores han determinado cuáles son los retos y las limitaciones que obstaculizan los esfuerzos para vincular la investigación con las acciones de política a nivel de país. ${ }^{4}$ Estos retos pueden agruparse en tres grandes categorías: el clima sociopolítico en cuanto al uso de las investigaciones, el proceso de producción de las investigaciones y la traslación o traducción de los resultados de las investigaciones. A menudo, se subestima el valor de la investigación, se la considera irrelevante o se cree que es demasiado difícil usarla para fundamentar la formulación de políticas.

Aunque en muchos países del continente los responsables de formular políticas reconocen los beneficios de usar la investigación como guía al tomar decisiones para mejorar los sistemas de salud, las medidas actuales son insignificantes en comparación con los logros del movimiento en pro de la medicina basada en la evidencia. Los responsables de producir investigaciones y los usuarios de estas investigaciones aun no logran sentar las bases científicas de la investigación sobre políticas y sistemas de salud. ${ }^{5}$ Además, aún no se ha establecido formalmente el uso sistemático de las investigaciones sobre los sistemas de salud en los procesos de formulación de políticas y, en la actualidad, este uso depende en gran medida de la experiencia previa de los responsables de tomar las decisiones y su relación personal con los investigadores y los profesionales de la atención de salud. ${ }^{6}$

En los países de la Región de las Américas, incluido el Caribe, se produce $46 \%$ de las investigaciones en salud pública que se realizan en el mundo. Sin embargo, la mayor parte $(37 \%)$ proviene de Estados Unidos ${ }^{7}$ y no siempre es pertinente para los Estados Miembros de la OPS. ${ }^{8}$ Se necesita un enfoque más sistemático para establecer las prioridades y financiar investigaciones que sean pertinentes y útiles para la Región. Contar con sistemas, personas, instituciones $\mathrm{y}$ actividades dedicadas a la investigación en materia de salud a nivel nacional que realicen investigaciones de buena calidad con el fin de promover, recuperar y mantener el estado de salud de la población es una manera de garantizar que la investigación satisfaga las necesidades de salud pública de un país. Sin embargo, en los países de la Región de las Américas el crecimiento y la consolidación de estos sistemas ha sido desigual, y se ha basado en iniciativas puntuales o aisladas de fortalecimiento de la capacidad. ${ }^{9}$

En el 2005, la Asamblea Mundial de la Salud instó a los países a establecer la infraestructura nacional necesaria para promover políticas basadas en la evidencia, así como sistemas de salud pública y de prestación de servicios de salud basados en la evidencia. ${ }^{10}$ En la práctica, esta infraestructura está conformada por programas, intervenciones y herramientas que difunden y facilitan el acceso a la información procedente de la investigación, fomentan el intercambio de conocimientos entre los interesados directos, y emplean los datos científicos para mantener informadas a las entidades que prestan servicios de salud y a los sistemas de salud. ${ }^{11}$ Sin embargo, muchos países de la Región de las Américas aún no cuentan con este tipo de infraestructura formal. ${ }^{12}$ En la mayoría de los casos, las actividades se realizan como proyectos puntuales que no cuentan con financiamiento sostenible más allá del ciclo de vida del proyecto. Aunque algunas iniciativas han promovido el diálogo entre quienes producen y quienes utilizan la investigación, la alta tasa de rotación de las autoridades de salud pública, e incluso dentro de los equipos técnicos de los sectores de la salud y la tecnología, obstaculizan las actividades a largo plazo de traducción del conocimiento. ${ }^{12}$ Además, la falta de una infraestructura formal para coordinar los esfuerzos nacionales para que las investigaciones se traduzcan en políticas y para que se promueva el intercambio de experiencias entre los países de la Región ha limitado el aprendizaje colectivo y el intercambio de recursos en el área de la traducción del conocimiento.

\section{INICIATIVAS EXITOSAS}

En la Región de las Américas no se ha realizado un examen exhaustivo sobre las iniciativas de traducción del conocimiento. Sin embargo, en Brasil, Chile y Perú hay casos en los cuales se han abordado los retos que se enfrentan cuando se intenta establecer un vínculo entre la investigación y las acciones de política. Los estudios de casos que se presentan a continuación fueron seleccionados por los autores debido al conocimiento directo que tenían de ellos.

En Perú, el clima sociopolítico para el uso de la investigación ha mejorado en los últimos años gracias a la labor de unidades de investigación no académicas que promueven el uso de la evidencia procedente de la investigación en el sistema de salud. Después de dos iniciativas en las cuales los resultados de varias investigaciones ayudaron a fundamentar algunas decisiones en materia de políticas, el Ministerio de Salud de Perú estableció procedimientos formales para solicitar evidencia generada a partir de la investigación. Las dos iniciativas giraban en torno a los efectos abortivos de la píldora anticonceptiva del día después y los efectos para la salud de los alimentos transgénicos. ${ }^{13}$ En la actualidad, las decisiones sobre los presupuestos de los programas, el rediseño de los programas de salud a nivel nacional y subnacional, y la selección de las intervenciones de salud específicas que financiará el ministerio deben basarse en la evidencia producto de la investigación. Para abordar la necesidad de contar con evidencia científica en Perú, se estableció la Unidad de Análisis y Generación de Evidencias en Salud Pública en el Centro Nacional de Salud Pública. ${ }^{14}$ Además, estos procesos formales se han ampliado a otras direcciones del Ministerio de Salud, y diversos grupos que trabajan en directrices clínicas y en la evaluación de tecnologías sanitarias han comenzado a colaborar.

En Chile, el Fondo Nacional de Investigación y Desarrollo en Salud (FONIS) es una iniciativa costo-eficaz que se estableció con el objetivo de producir investigaciones pertinentes y ayudar a los responsables de tomar decisiones a abordar los problemas del sistema de salud del país. ${ }^{15}$ Desde el 2003, el FONIS ha financiado proyectos seleccionados por medio de convocatorias competitivas en las áreas de eficacia de las intervenciones clínicas y de salud pública; salud ambiental y ocupacional; equidad en el acceso a la atención de salud; promoción de la salud y control de factores de riesgo; calidad de la atención de salud y satisfacción de los usuarios de los servicios de salud; y métodos para determinar el impacto en la 
Recuadro 1. La experiencia de EVIPNet-Brasil

- Impulsada por las altas tasas de mortalidad en menores de 1 año en las regiones norte y noreste de Brasil, en el 2016 EVIPNet-Brasil publicó una serie de ocho resúmenes de evidencia de salud para políticas en áreas prioritarias clave para la salud pública. Los datos científicos indicaron que había una correlación directa entre la elevada mortalidad en menores de 1 año y las deficiencias en la calidad de la atención durante el parto, con trabajadores de salud insuficientemente capacitados que no seguían las directrices y los protocolos establecidos para la atención de salud. En el municipio de Piripiri, de bajos recursos, la investigación mostró que $60 \%$ de las muertes de recién nacidos podrían haberse evitado.

- EVIPNet-Brasil, en alianza con el consejo local de salud, abordó el problema presentando diversas opciones de política fundamentadas en la evidencia. Los diálogos sobre políticas llevaron a seleccionar las opciones que ofrecían el mayor impacto posible, lo que implicó un complejo proceso de planificación local de más de 30 intervenciones basadas en la mejor evidencia disponible. Esta labor ayudó a reducir las tasas de mortalidad en menores de 1 año en Piripiri de 21 por 1.000 nacidos vivos en el 2009 a 7 por 1.000 nacidos vivos en el 2011.

- Actualmente, EVIPNet-Brasil está adaptando esta evidencia para crear un modelo de política, de manera que pueda repetirse la experiencia en otras localidades brasileñas para que los grupos de trabajo locales aborden sus propios problemas de salud, preparen resúmenes de evidencia y organicen diálogos sobre políticas en torno a los problemas que tienen. Entre otros asuntos prioritarios de salud pública se encuentran la anemia de células falciformes, la contaminación del aire, las cardiopatías congénitas, la tuberculosis en las personas sin hogar, el desarrollo del niño en la primera infancia y las muertes causadas por el tránsito.

salud de las políticas públicas ejecutadas por sectores distintos al de la salud. En sus primeros diez años, el FONIS financió 300 proyectos con un costo total de alrededor de $\$ 10$ millones ( $£ 7,5$ millones; $€ 8,6$ millones). Además, las investigaciones financiadas por el FONIS han tenido impacto en la política nacional: los cambios en la ley sobre el consumo de tabaco en Chile, por ejemplo, se fundamentaron en una investigación financiada por el FONIS. ${ }^{16} 17$

En el 2006, la OMS estableció la Red de Políticas Basadas en la Evidencia (EVIPNet) para abordar el reto de traducir la investigación en políticas en todo el mundo, incluida la Región de las Américas. EVIPNet establece alianzas sostenibles entre los responsables de formular políticas, los investigadores y la sociedad civil para aumentar la capacidad de los países de elaborar políticas de salud usando evidencia producto de la investigación. ${ }^{18}$ La OPS estableció EVIPNet en la Región de las Américas en el 2007 y, para fines del 2013, doce países contaban con equipos de esta red..$^{12}$ Estos equipos a nivel de país forman la infraestructura institucional necesaria para traducir los resultados de la investigación en políticas eficaces. Los dos productos principales de estos equipos son resúmenes de evidencia para políticas, en los cuales se presenta una síntesis contextualizada y fácil de usar de las diferentes opciones en cuanto a las políticas, y los diálogos sobre políticas, que son debates estructurados centrados en un resumen de evidencia y se usan para formular políticas. ${ }^{19}$ Para el 2013, se había impartido capacitación a más de 700 funcionarios y expertos a nivel de país, se habían elaborado catorce resúmenes de evidencia y se habían realizado diez diálogos sobre políticas en ocho países. ${ }^{12}$ Brasil ha liderado la puesta en práctica exitosa de las actividades y los productos de EVIPNet (véase el recuadro 1)..$^{20}$ Aunque esta red no es la única iniciativa emprendida para abordar el reto de traducir la investigación en políticas, ha servido de modelo para organizar actividades en otros países del continente como Chile ${ }^{21}$ y ha generado oportunidades en el terreno para aumentar la capacidad, al brindar asistencia a investigadores y responsables de tomar decisiones por medio de la red mundial de la iniciativa EVIPNet.

\section{AGENDA PENDIENTE}

Para producir investigación pertinente que permita fundamentar la toma de decisiones en el ámbito de la salud, los países tienen que adoptar un enfoque sistemático con el fin de fortalecer los sistemas nacionales de investigación en salud. ${ }^{22}$ Para ello se requiere una política o un programa bien definido vinculado a las prioridades nacionales en cuanto a la investigación para la salud; estructuras para coordinar y gestionar las investigaciones; mecanismos de financiamiento sostenibles; y un conjunto definido de indicadores para las actividades de seguimiento y evaluación. La OPS está en posición de promover la adopción de este enfoque en los países de la Región de las Américas, a fin de aprovechar las experiencias exitosas y conectar sistemáticamente las distintas iniciativas que actualmente funcionan de manera aislada.

A pesar de que la Región de las Américas ha tenido cierto éxito en cuanto a la traducción del conocimiento, ${ }^{20} 21$ la mayor parte de los países debe fortalecer la infraestructura necesaria para hacerlo. Además, los países de la Región tienen que promover la creación de equipos dedicados a poner en práctica la investigación y brindarles apoyo. Esto es crucial para aquellos países donde se han llevado a cabo algunas actividades de este tipo, pero no se ha establecido ninguna infraestructura formal de colaboración regional o mundial. Más allá de los problemas de financiamiento, analizar los éxitos y los fracasos después de diez años de iniciativas en este campo es una de las prioridades a corto plazo. La OPS se ha propuesto enfrentar los retos que obstaculizan la traducción del conocimiento por medio de la colaboración estrecha con los asociados y de alianzas estratégicas, como la colaboración con la Universidad McMaster (de Canadá) y el Centro Latinoamericano y del Caribe de Información en Ciencias de la Salud (de Brasil), la disponibilidad de la Biblioteca Virtual en Salud y el amplio sistema regional de bases de datos bibliográficas, y la colaboración con la Alianza para la Investigación en Políticas y Sistemas de Salud de la OMS.

En ningún país de la Región de las Américas se ha realizado una evaluación formal del clima sociopolítico en torno al uso de la investigación para fundamentar políticas; los datos a este respecto se encuentran esparcidos en diversos 
documentos de política de diferentes países. Tampoco se ha hecho un esfuerzo sistemático para recopilar información acerca de las actitudes predominantes con respecto a la traducción del conocimiento, como las iniciativas que se han llevado a cabo en el Oriente Medio y algunos países de África. ${ }^{23}{ }^{24}$ Este tipo de información podría facilitar el diseño de estrategias específicas. La OPS tiene la posibilidad de movilizar a grupos de expertos regionales que trabajan en el campo y abordar este tema.

\section{CONCLUSIONES}

A nivel de país, subsisten algunos retos para establecer un vínculo entre la investigación y las acciones de política, que se relacionan con el clima sociopolítico en torno al uso de la investigación, la producción de investigaciones pertinentes, y la aplicación de los resultados de la investigación. La Política de investigación para la salud de la OPS ha proporcionado un marco general para establecer iniciativas exitosas en la Región de las Américas. En el último decenio, se han logrado avances en cuanto al establecimiento de sistemas nacionales de investigación en salud y la disponibilidad de equipos a nivel de país con las aptitudes necesarias para definir, evaluar, resumir y organizar la evidencia producto de la investigación. También se han logrado avances en el establecimiento de procesos estandarizados para el uso de la evidencia en la formulación de políticas (por ejemplo, los procedimientos de la EVIPNet) y se cuenta con equipos de expertos regionales que brindan apoyo a estas iniciativas. Sin embargo, subsisten obstáculos en los sistemas de salud de la Región que impiden que las investigaciones tengan un impacto. La OPS y su Política de investigación para la salud desempeñarán un papel clave en la definición de los próximos pasos para lograr que la investigación sobre los sistemas de salud en la Región de las Américas tenga un impacto positivo en la salud.

Agradecimientos. Queremos expresar nuestro agradecimiento a Luis Gabriel Cuervo y Luis Alejandro Salicrup, de la Oficina de Gestión del Conocimiento, Bioética e Investigación, OPS/OMS; y a Lisa Bero, de la Universidad de Sydney.

Contribución de los autores. TP es médico de familia, profesor universitario e investigador del sistema de salud. JB tuvo una posición de autoridad en el Municipio de Piripiri, en la región noreste de Brasil, y fue coordinador nacional de EVIPNet-Brasil en el Ministerio de Salud de ese país. UP trabajó como funcionario de alto nivel de la OMS en Ginebra, donde coordinó la secretaría mundial de la EVIPNet. El presente artículo se basa en estudios de casos publicados en revistas arbitradas, documentos de política y la experiencia de los autores como investigadores y responsables de la toma de decisiones a diferentes niveles del sistema de salud. Todos los autores han contribuido con el artículo y coinciden

\section{REFERENCIAS}

1. Declaración de México sobre las investigaciones sanitarias. Conocimientos para una mejor salud: fortalecimiento de los sistemas de salud. Cumbre Ministerial sobre Investigación en Salud. 2004. https:// www.who.int/rpc/summit/agenda /Mexico_Statement-Spanish.pdf.

2. Lancet. The Bamako call to action: research for health. Lancet 2008;372:1855. doi:10.1016/S0140-6736(08)61789-4

3. Organización Panamericana de la Salud. Política de investigación para la salud. Documento CD49/10. 2009. https:// www.paho.org/hq/images/stories/KBR /Research/politica\%20de\%20investiga cion $\% 20$ para $\% 201$ \% $\% 20$ salud.pdf?ua $=1$.

4. Lavis JN, Lomas J, Hamid M, Sewankambo NK. Assessing country-level efforts to link research to action. Bull World Health Organ 2006;84:620-8. doi:10.2471/BLT.06.030312

5. Bennett S, Agyepong IA, Sheikh K, Hanson K, Ssengooba F, Gilson L. Building the field of health policy and systems research: an agenda for action. PLoS Med 2011;8:e1001081.doi:10.1371/journal.pmed. 1001081

6. Oliver K, Innvar S, Lorenc T, Woodman J, Thomas J. A systematic review of barriers to and facilitators of the use of evidence by policymakers. BMC Health Serv Res 2014;14:2. doi:10.1186/1472-6963-14-2

7. Zacca-González G, Chinchilla-Rodríguez Z, Vargas-Quesada B, de Moya-Anegón F. Bibliometric analysis of regional Latin America's scientific output in Public Health through SCImago Journal \& Country Rank. BMC Public Health 2014;14:632. doi:10.1186/1471-2458-14-632

8. Reveiz L, Sangalang S, Glujovsky D, et al. Characteristics of randomized trials published in Latin America and the Caribbean according to funding source. PLoS One 2013;8:e56410. doi:10.1371/journal.pone. 0056410 en su contenido; TP es el garante del artículo. Las opiniones expresadas son de los autores y no reflejan necesariamente la posición de las instituciones a las que están afiliados.

Conflicto de intereses. Hemos leído y comprendido la política de The BMJ sobre declaración de intereses y no tenemos intereses pertinentes que declarar.

Procedencia del artículo y revisión por pares. Comisionado; revisión externa por pares.

Declaración. Las opiniones expresadas en este manuscrito son responsabilidad del autor y no reflejan necesariamente los criterios ni la política de la RPSP / PAJPH y/o de la OPS/OMS.

Serie Fortalecimiento de la investigación para la salud en las Américas. Este artículo es parte de una serie propuesta por la Organización Panamericana de la Salud (OPS) y comisionada por The BMJ, que tuvo a cargo la revisión por pares y la edición, y tomó la decisión sobre la publicación de los artículos sin participación de la OPS. Los gastos de publicación en acceso abierto de la serie original en inglés fueron financiados por la OPS. Acceso a la serie original en inglés en www.bmj.com /health-research-americas; accesoalaserie en español y portugués en https: / / www. paho.org/journal/es/numeros-especiales / fortalecimiento-investigacion-para-sa lud-americas.
9. Becerra-Posada F, Minayo M, Quental C, de Haan S. National research for health systems in Latin America and the Caribbean: moving towards the right direction? Health Res Policy Syst 2014;12:13. doi: 10.1186/1478-4505-12-13

10. Organización Mundial de la Salud. 58. ${ }^{a}$ Asamblea Mundial de la Salud: resoluciones y decisiones. 2005. http://apps.who .int/gb/ebwha/pdf_files/WHA58-REC1 /A58_2005_REC1-sp.pdf.

11. Ellen ME, Lavis JN, Ouimet M, Grimshaw J, Bédard P-O. Determining research knowledge infrastructure for healthcare systems: a qualitative study. Implement Sci 2011;6:60. doi:10.1186/1748-5908-6-60

12. Chapman E. Management report and evaluation of EVIPNet Americas 2010-2013. Organización Panamericana de la Salud. 2013. www.paho.org/hq/index.php?op tion $=$ com_docman\&task=doc_view \&Itemid $=270 \&$ gid $=31200 \& l a n g=e n$. 
13. Entrevista a Oscar Ugarte Ubilluz, ministro de Salud de Perú, acerca del uso de políticas basadas en la evidencia para la toma de decisiones. 2011. www.paho.org /hq/index.php?option=com_content\& view $=$ article \&id $=5623 \&$ Itemid $=3970$

14. Gutierrez EL, Piazza M, Gutierrez-Aguado A, et al. Uso de la evidencia en políticas y programas de salud aportes del instituto nacional de salud. Rev Peru Med Exp Salud Publica 2016;33:580-4. doi:10.17843/ rpmesp.2016.333.2308

15. Fondo Nacional de Investigacion en Salud. 10 años apoyando la investigación aplicada en salud en Chile. 2014. www. conicyt.cl/fonis / files / 2014/12/Fonis-10 -anios-de-apoyando-la-investigaci $\%$ C3\% B3n-aplicada-en-salud-en-Chile.pdf.

16. Iglesias V, Erazo M, Droppelmann A, et al. Occupational second hand smoke is the main determinant of hair nicotine concentrations in bar and restaurant workers. Environ Res 2014;132:206-11. doi:10.1016/j. envres.2014.03.044

17. República de Chile. Ministerio de Salud. Ley 20.660: Modifica ley N. ${ }^{\circ}$ 19.419, en materia de ambientes libres de humo de tabaco. 2013. www.leychile.cl/Navegar?id Norma $=1047848$.

18. Organización Mundial delaSalud.EVIPNet in action: 10 years, 10 stories. 2016. www. who.int/evidence/resources/publica tion/en.

19. Moat KA, Lavis JN, Clancy SJ, El-Jardali F, Pantoja T. Knowledge Translation Platform Evaluation study team. Evidence briefs and deliberative dialogues: perceptions and intentions to act on what was learnt. Bull World Health Organ 2014;92:20-8. doi:10.2471/BLT.12.116806

20. Wichmann R, Carlan E, Barreto J. Consolidation for evidence-informed policy network: EVIPNet Brazil: report of the national experience of building a knowledge translation platform for Brazilian Public Healthcare System (SUS). Bol Inst Saude 2016;17:13-25.

21. Mansilla C, Herrera CA, Basagoitia A, Pantoja T. The evidence-informed policy network in Chile: lessons learned from a year of coordinated efforts. Rev Panam Salud Publica 2017:41:e36.

22. Erazo A. Un enfoque sistémico para comprender y mejorar los sistemas de salud. Rev Panam Salud Publica 2015;38 248-53.

23. El-Jardali F, Ataya N, Jamal D, Jaafar M. A multi-faceted approach to promote knowledge translation platforms in eastern Mediterranean countries: climate for evidence-informed policy. Health Res Policy Syst 2012;10:15. doi:10.1186/1478 $-4505-10-15$

24. Ongolo-Zogo P, Lavis JN, Tomson G, Sewankambo NK. Climate for evidence informed health system policymaking in Cameroon and Uganda before and after the introduction of knowledge translation platforms: a structured review of governmental policy documents. Health Res Policy Syst 2015;13:2. doi:10.1186/1478-4505-13-2

Forma de citar (artículo original): BMJ 2018;362:k2469 doi: http:/ /dx.doi.org/10.1136/bmj.k2469

Manuscrito original en inglés publicado el 16 de julio del 2018. 